\title{
Electronic interactions in illuminated carbon dots/MoS ensembles and electrocatalytic activity towards hydrogen evolution
}

\author{
Ruben Canton-Vitoria ${ }^{[\mathrm{a}] \#}$, Lorenzo Vallan ${ }^{[\mathrm{b}] \#}$, Esteban Urriolabeitía ${ }^{[\mathrm{c}]}$, Ana M. Benito ${ }^{[\mathrm{b}]}$, Wolfgang K. Maser ${ }^{[\mathrm{b}]}$, \\ Nikos Tagmatarchis*[a]
}

\begin{abstract}
We report on the preparation, characterization and photophysical and electrocatalytic properties of carbon dots (CDs)/MoS ${ }_{2}$ ensembles. Based on electrostatic interactions, ammonium functionalized $\mathrm{MoS}_{2}$, prepared upon reaction of 1,2dithiolane tert-butyl carbamate with $\mathrm{MoS}_{2}$ followed by acidic deprotection, was coupled with CDs bearing multiple carboxylates on their periphery as derived upon microwave-assisted polycondensation of citric acid and ethylenediamine followed by alkaline treatment. Insights into electronic interactions between the two species within $\mathrm{CDs} / \mathrm{MoS}_{2}$ emanated from absorption and photoluminescence titration assays. Efficient fluorescence quenching of CDs by $\mathrm{MoS}_{2}$ was observed and attributed to photoinduced electron/energy transfer as the decay mechanism for the transduction of the singlet excited state of CDs. Finally, the electrocatalytic performance of $\mathrm{CDs} / \mathrm{MoS}_{2}$ was assessed towards the hydrogen evolution reaction and found superior as compared to that owed to the individual CDs species.
\end{abstract}

\section{Introduction}

One of the most actively investigated 2D materials beyond graphene is arguably $\mathrm{MoS}_{2}$, which belongs to the larger family of layered transition metal dichalcogenides (LTMDCs). ${ }^{[1]} \mathrm{A}$ single layered $\mathrm{MoS}_{2}$ is a semiconducting pseudo-2D crystal consisting of a metal network sandwiched within two chalcogenide layers, where each metal cation is connected with four chalcogenide anions forming a honeycomb lattice. Bulk LTMDCs can be exfoliated to semiconducting or metallic sheets depending on the exfoliating agents employed. Notably, we recently developed a facile approach leading to exfoliated semiconducting LTMDCs via treatment with chlorosulfonic acid, ${ }^{[2]}$ contrasting the BuLi

\footnotetext{
[a] R. Canton-Vitoria, N. Tagmatarchis Theoretical and Physical Chemistry Institute National Hellenic Research Foundation 48 Vassileos Constantinou Avenue, 11635 Athens, Greece. E-mail: tagmatar@eie.gr

[b] L. Vallan, A. M. Benito, W. K. Maser Instituto de Carboquímica (ICB, CSIC) Consejo Superior de Investigaciones Científicas C/Miguel Luesma Castán 4, E-50018 Zaragoza, Spain.

[c] Esteban Urriolabeitía

Instituto de Síntesis Quimica y Catálisis Homogénea CSIC, Universidad de Zaragoza

* C/Pedro Cerbuna 12, E-50009 Zaragoza, Spain Equal contribution.

Supporting information for this article is given via a link at the end of the document.
}

treatment exfoliation process that gives access predominantly to the metallic polytype structure. ${ }^{[3]}$

A major drawback of LTMDCs materials is associated with poor solubility, since multiple van der Waals forces between the layers keep them tightly together. Those forces, preventing the separation and individualization of sheets within the LTMDCs, can be overcome by chemical functionalization and the incorporation of species that due to steric and/or electronic reasons may keep apart, by avoiding re-stacking, the sheets of LTMDCs. Hence, functionalization of LTMDCs is imperative for fully harnessing their capabilities and broaden their application in a variety of fields. However, LTMDCs tend to be rather inert chalcogen atoms in the basal plane are saturated and not highly reactive, while the metal atoms are embedded beneath the chalcogen layer, thus not susceptible to functionalization. Nevertheless, reactions of metallic $\mathrm{MoS}_{2}$ with organoiodides or diazonium salts yielded $\mathrm{MoS}_{2}$-based materials carrying organic addends at the basal plane. ${ }^{[4]}$ Moreover, ligand conjugation of thiols to Mo atoms of metallic $\mathrm{MoS}_{2},{ }^{[5]}$ as a rather debatable route of functionalization, since thiols can convert to the corresponding disulfides in a catalytic reaction activated by the presence of $\mathrm{MoS}_{2}$, which eventually physisorb onto the basal plane of $\mathrm{MoS}_{2},{ }^{[6]}$ was also reported. In addition, we recently succeeded the covalent functionalization of exfoliated semiconducting $\mathrm{MoS}_{2}$, by exploiting the high binding affinity of 1,2-dithiolanes for Mo atoms, particularly those located at the edges of exfoliated semiconducting $\mathrm{MoS}_{2}$, where $\mathrm{S}$ vacancy sites are naturally introduced during the chemical exfoliation from the bulk. ${ }^{[7]}$

Carbon dots (CDs), since their serendipitously discovery in $2004,{ }^{[8]}$ proved to be a valuable new entry in the horizon of carbon-based nanomaterials. In general, CDs are nanosized particles, with diameter in the order of $10 \mathrm{~nm}$ or less, showing solubility in aqueous and organic media, ${ }^{[9]}$ biocompatibility ${ }^{[10]}$ and photochemical stability. ${ }^{[11]}$ Among the novel physicochemical properties of CDs is their intrinsic photoluminescence, ${ }^{[12]}$ which can be exploited in energy conversion schemes. ${ }^{[13]}$ In particular, photoinduced energy and/or electron transfer processes in CDs have been identified, for example, when combined with carbon nanotubes ${ }^{[14]}$ graphene oxide, ${ }^{[15]}$ perylene diimides, ${ }^{[16]}$ and porphyrins. ${ }^{[17]}$ Moreover, the high stability and good electrical conductivity of CDs enable them to be considered as interesting contenders for electrocatalysis. ${ }^{[18]}$

Considering all the above issues, the aim of the current study is three-fold, namely to (i) integrate, by employing electrostatic attractive forces, $\mathrm{MoS}_{2}$ and $\mathrm{CDs}$ possessing opposite charges, into an electron donor-acceptor $\mathrm{CDs} / \mathrm{MoS}_{2}$ system, (ii) scrutinize intra-ensemble electronic interactions 
between the two species, and (iii) assess the electrocatalytic performance of $\mathrm{CDs} / \mathrm{MoS}_{2}$ towards the hydrogen evolution reaction (HER). To achieve these goals, semiconducting $\mathrm{MoS}_{2}$ sheets, as obtained upon wet-exfoliation from the bulk, were functionalized such as to carry positively charged ammonium moieties, and combined with CDs bearing carboxylate anions. Electronic absorption spectroscopy as well as steady-state and time-resolved photoluminescence titration assays were employed to shed light on the electronic communication and the dynamics of the photoinduced processes developed within the $\mathrm{CDs} / \mathrm{MoS}_{2}$ ensembles. Finally, overpotentials and Tafel slopes were evaluated for the assessment of the electrocatalytic activity of $\mathrm{CDs} / \mathrm{MoS}_{2}$ toward the HER.

\section{Results and Discussion}

Bulk $\mathrm{MoS}_{2}$ was exfoliated to semiconducting nanosheets following the chlorosulfonic acid assisted strategy. ${ }^{[2]}$ The exfoliated $\mathrm{MoS}_{2}$ was spectroscopically evaluated and found to be around $340 \mathrm{~nm}$ in lateral size and oligolayered thick. ${ }^{[19]}$ Treatment of exfoliated $\mathrm{MoS}_{2}$ with the 1,2-dithiolane tert-butyl carbamate (BOC) derivative $\mathbf{1}$, furnished material 2 , according to Figure 1a. This involves the recently established methodology for the functionalization of chemically-exfoliated LTMDCs, based on the high binding affinity of 1,2-dithiolanes for the Mo atoms, ${ }^{[7]}$ particularly those located at the edges of exfoliated $\mathrm{MoS}_{2}$. Filtration of the reaction mixture through a PTFE membrane with

(a)

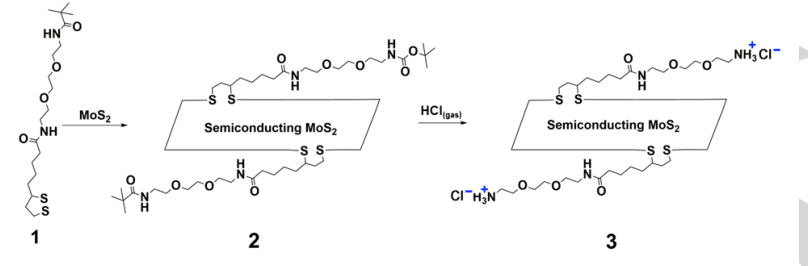

(b)

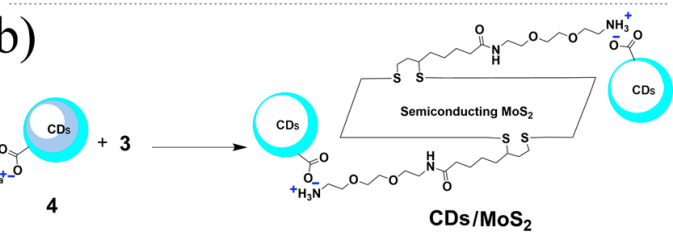

Figure 1. (a) Functionalization of $\mathrm{MoS}_{2}$ leading to ammonium modified $\mathrm{MoS}_{2}$-based material 3. (b) Formation of $\mathrm{CDs} / \mathrm{MoS}_{2}$ ensembles based on electrostatic interactions.

$0.2 \mu \mathrm{m}$ pore size and successive washing with dichloromethane guaranteed that modified $\mathrm{MoS}_{2}$ material 2 was free of non- covalently associated organic species. The presence of the ethylene glycol side chain in $\mathbf{2}$ allowed solubility enhancement, tolerating easier handling and manipulation in wet media. Furthermore, the BOC terminating group in 2, masking the introduced amino-functions, can be easily removed under acidic conditions, yielding ammonium functionalized $\mathrm{MoS}_{2}$ material 3 .

The chemical transformation was spectroscopically proved by observing alterations in the ATR-IR spectra of materials $\mathbf{2}$ and $\mathbf{3}$. While stretching vibration bands due to $\mathrm{C}-\mathrm{H}$ units are identified in the region 2800-3000 $\mathrm{cm}^{-1}$ for both 2 and 3, two discrete bands at 1650 and $1710 \mathrm{~cm}^{-1}$ owed to carbonyl amide and BOC units, respectively, are present in the IR spectrum of 2 , with the latter band being absent in the spectrum of 3 (Supporting Information, Figure S1), thus justifying the effective BOCdeprotection. Raman spectroscopy revealed the presence of characteristic $A_{1 g}$ and $E_{2 g}^{1}$ modes located at 406 and $382 \mathrm{~cm}^{-1}$, respectively, in materials 2 and 3 . Moreover, the $A_{1 g}$ and $E_{2 g}^{1}$ modes were found unaltered as compared with the ones present in exfoliated $\mathrm{MoS}_{2}$ (Supporting Information, Figure S2). Since for exfoliated $\mathrm{MoS}_{2}$ the calculated frequency difference between $\mathrm{A}_{1 \mathrm{~g}}$ and $E_{2 g}^{1}$ is $24 \mathrm{~cm}^{-1}$, corresponding to the presence of 3-4 MoS layers in average ${ }^{[20]}$ it is reasonable to claim that the same number of layers exists in $\mathbf{2}$ and $\mathbf{3}$. Additionally, no other Raman bands were observed in the region $500-1000 \mathrm{~cm}^{-1}$, indicating the absence of oxidation during the exfoliation and functionalization process, hence, proving the preservation of the electronic properties of the semiconducting $\mathrm{MoS}_{2}$ polytype. In addition, the $\zeta$-potential value changed from $-24 \mathrm{mV}$ for exfoliated $\mathrm{MoS}_{2}$ to $+2.6 \mathrm{mV}$ for 3 , being consistent with the presence of ammonium functionalities. Moreover, Kaiser test revealed a value of 50 $\mu \mathrm{mol} / \mathrm{g}$ for free amine units in 3 . Then, based on TGA analysis, the $4.5 \%$ mass loss observed during heating of 3 in the temperature range $200-500{ }^{\circ} \mathrm{C}$ under nitrogen atmosphere (Supporting Information, Figure S3), related to the decomposition of the organic part incorporated on $\mathrm{MoS}_{2}$, is consistent with the presence of one functional group for every 49 units of $\mathrm{MoS}_{2}$. As far as morphology concerns, the $\mathrm{MoS}_{2}$ material 3 was examined by field-emission SEM. Briefly, a few drops of a methanol dispersion of $\mathbf{3}$ were deposited onto the sample holder and imaged after the solvent was allowed to slowly evaporate. Polygonal overlapping sheets of $\mathrm{MoS}_{2}$ with sizes varied between hundreds of nanometers to several micrometers in a random distribution were observed (Supporting Information, Figure S4). Amplification of several areas of the material reveals semi-transparent sheets associated to few layers of $\mathrm{MoS}_{2}$, with regular and linear edges. It should be pointed out that due to the drying process for imaging, re-staking of the $\mathrm{MoS}_{2}$ layers occurs explaining not only the deviation observed from the spectroscopically calculated layer size as in solution but also the avoidance of identifying single-layered $\mathrm{MoS}_{2}$ in the modified material 3. 
In parallel, CDs were synthesized from citric acid and ethylenediamine following a microwave-assisted
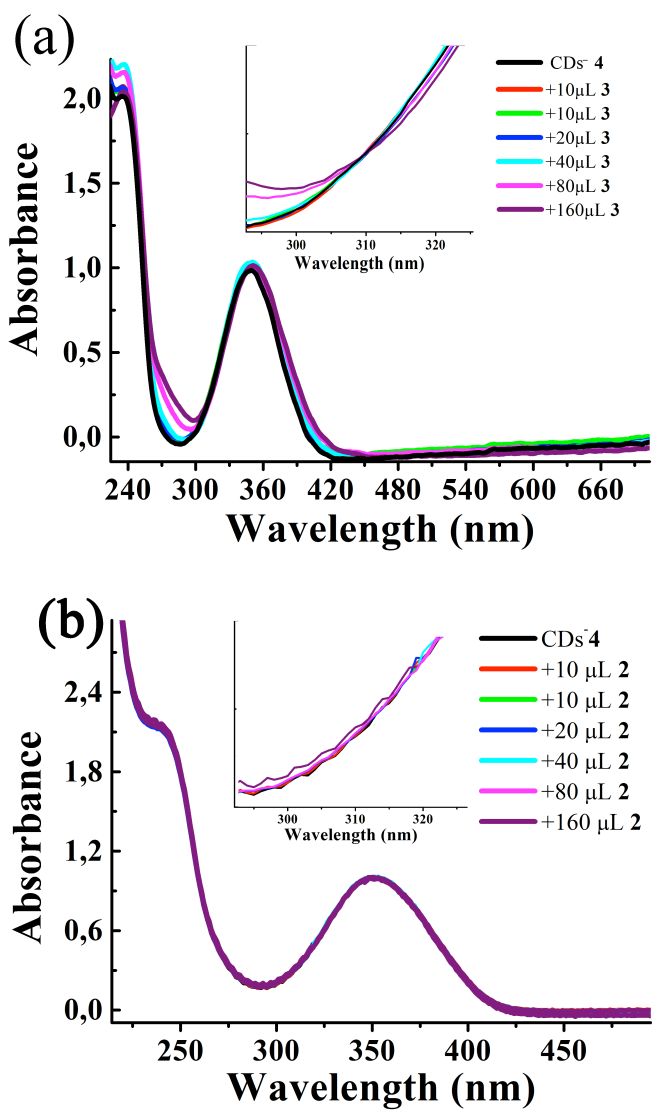

Figure 2. UV-Vis absorption spectra of $\mathrm{CDs}^{-} \mathbf{4}$ upon incremental additions of (a) ammonium modified $\mathrm{MoS}_{2}$-based material 3. Inset: Enlargement of the $300-320 \mathrm{~nm}$ region where the isosbestic point is developed, (b) $\mathrm{MoS}_{2}$-based material 2.

polycondensation method. The microwave irradiation conditions employed guaranteed fast and easy production of 1-2 $\mathrm{nm}$ sized fluorescent CDs as revealed by DLS and AFM measurements (Supporting Information, Figure S5), possessing a polyamidic structure with a periphery rich in carboxylic acid moieties as verified by NMR (Supporting Information, Figure S6) and IR assays (Supporting Information, Figure S7). The plethora of $\mathrm{COOH}$ units decorating the surface of the water-soluble CDs can be easily ionized upon addition of aqueous $\mathrm{NaOH}$, giving access to negatively charged $\mathrm{CDs}^{-} 4$, which were further purified to remove the excess of $\mathrm{NaOH}$ by dialysis (molecular weight cutoff $0.5-1.0 \mathrm{kDa}$ ). As far as the optical properties of 4 concerns, they were screened by electronic absorption and photoluminescence spectroscopy. In brief, the UV-Vis spectrum of $\mathbf{4}$ is governed by two characteristic bands centred at 350 and $230 \mathrm{~nm}$ (Supporting Information, Figure S8), while upon $370 \mathrm{~nm}$ excitation an emission at $460 \mathrm{~nm}$ is identified (Supporting Information, Figure S9).

Next, having in hand modified $\mathrm{MoS}_{2}$ with positive charges in material 3, and negatively charged $C D s$ in material $\mathbf{4}$,
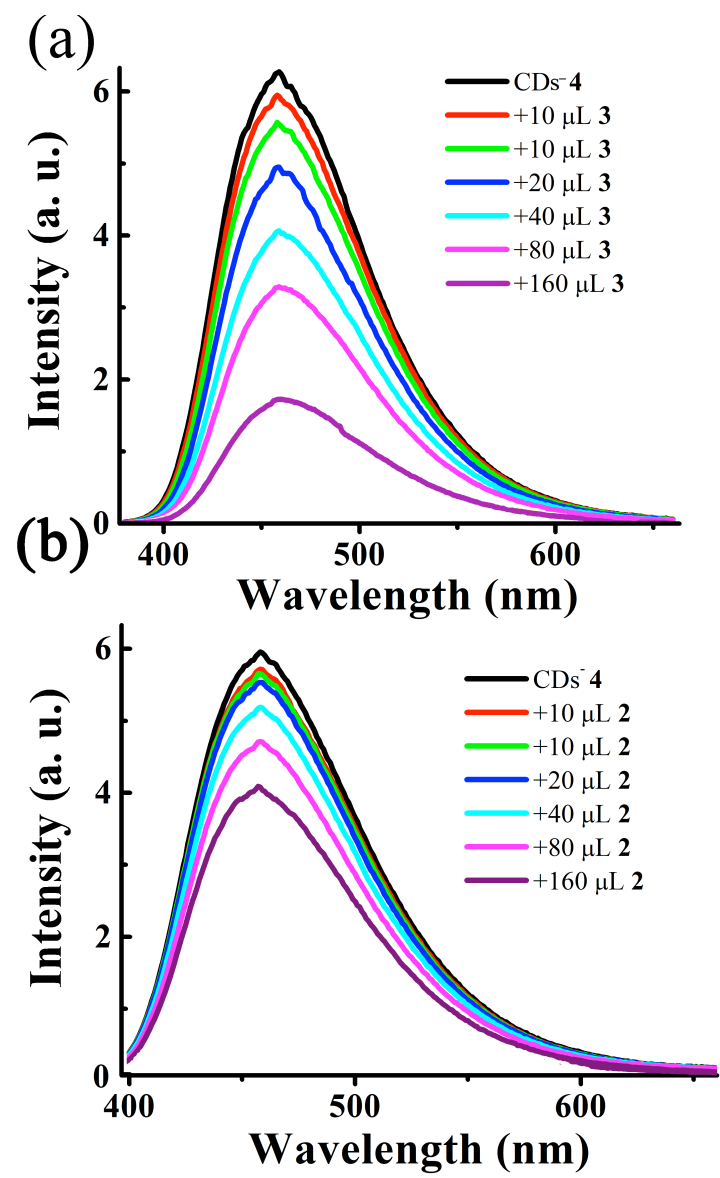

Figure 3. Photoluminescence titration assays of $\mathrm{CDs}^{-} 4(20 \mu \mathrm{g} / \mathrm{mL})$ upon incremental additions of (a) positively charged $\mathrm{MoS}_{2}$-based material 3 , and (b) neutral $\mathrm{MoS}_{2}$-based material 2. Measurements were conducted in water for samples possessing equal absorbance at the excitation wavelength of $370 \mathrm{~nm}$.

electrostatic attractive interactions between the two species were exploited (Figure 1b) en route the realization of $\mathrm{CDs} / \mathrm{MoS}_{2}$ ensembles. In this direction, a series of aqueous titration assays were conducted. Figure 2 shows the electronic absorption spectra of $\mathrm{CDs}^{-} 4$ upon addition of incremental amounts of ammonium modified $\mathrm{MoS}_{2} 3$. Subtraction of the $\mathrm{MoS}_{2}$ absorption background allowed a clear observation of the titration effects please note that $\mathrm{MoS}_{2}$ only shows characteristics bands beyond $490 \mathrm{~nm}$, i.e. beyond the absorption bands of $\mathrm{CDs}^{-} 4$ - and particularly the effect of $\mathrm{MoS}_{2}$ addition on the CDs 4 spectrum in UV-Vis titration assays without subtracting the $\mathrm{MoS}_{2}$ bands, the characteristic absorptions of the semiconducting phase of $\mathrm{MoS}_{2}$ appear at 690, 630 and $490 \mathrm{~nm}$ (Supporting Information, Figure S10). Evidently, a progressive red-shift for the absorption of $\mathrm{CDs}^{-} 4$ is observed, namely from 350 to $355 \mathrm{~nm}$ after the addition of $320 \mu \mathrm{L}$ of 3 . Moreover, a broad isosbestic point at $310 \mathrm{~nm}$, consistent with the complex formation between the two species in the ground state, was developed. Notably, when blank titration assays were conducted, by employing the neutral $\mathrm{MoS}_{2}$-based derivative 2 instead of the positively charged $\mathbf{3}$, 
(a)

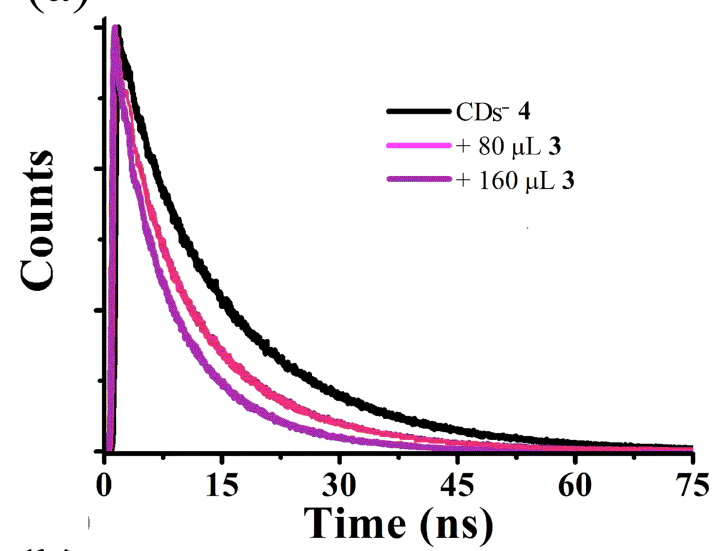

(b)

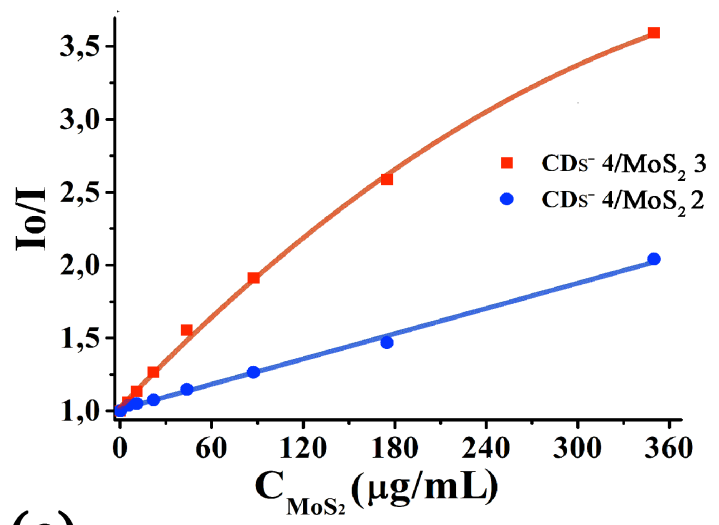

(c)

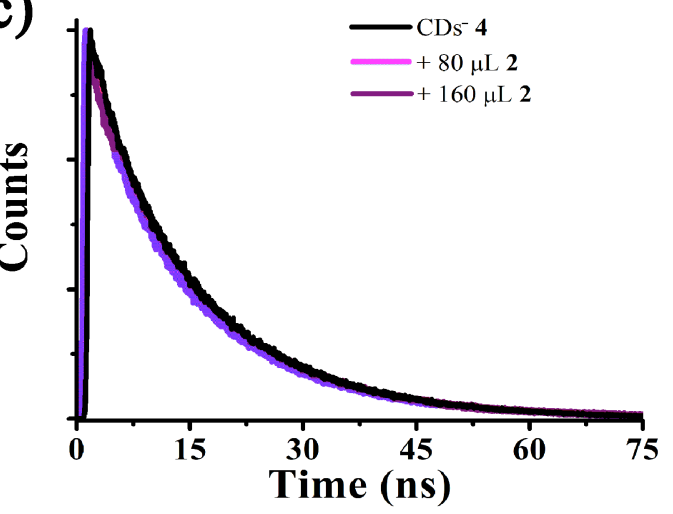

Figure 4. (a) Decay profiles for $\mathrm{CDs}^{-} 4$ upon incremental additions of positively charged $\mathrm{MoS}_{2}$-based material 3. (b) Stern-Volmer plot for the additions of the positively charged $\mathrm{MoS}_{2}$-based material 3 (red) and the neutral $\mathrm{MoS}_{2}$-based material 2 (blue) to $\mathrm{CDs}^{-} 4$. (c) Decay profiles for $\mathrm{CDs}^{-} \mathbf{4}$ upon incremental additions of neutral $\mathrm{MoS}_{2}$-based material 2.

none of the aforementioned results were observed (Figure 2b), thus, proving the beneficial role of attractive Coulombic forces not only in the establishment of the $\mathrm{CDs} / \mathrm{MoS}_{2}$ ensembles but also on the progression of effective interactions between $\mathrm{MoS}_{2}$ and CDs.
Meaningful insight on photoinduced electronic interactions between $\mathrm{MoS}_{2}$ and CDs at the excited states was derived from photoluminescence measurements. Steady-state assays revealed that the $460 \mathrm{~nm}$ fluorescence emission of $\mathrm{CDs}^{-} 4$, upon excitation at $370 \mathrm{~nm}$, was progressively quenched upon addition of the ammonium modified $\mathrm{MoS}_{2}$-based material 3 (Figure 3a), for samples possessing equal absorbance at the excitation wavelength. Notably, when blank assays were conducted, by incorporating the neutral $\mathrm{MoS}_{2}$-based derivative 2 instead of the positively charged $\mathbf{3}$, a lower quenching rate for the emission of $\mathrm{CDs}^{-} 4$ was observed (Figure $3 \mathrm{~b}$ ), ascribed to the inner filter effect of $\mathrm{MoS}_{2}$. These results suggest strong electronic interactions at the excited states between the positive and negative species within the $\mathrm{CDs} / \mathrm{MoS}_{2}$ ensemble. The photoluminescence quenching is supportive of photoinduced electron and/or energy transfer as the decay mechanism for the transduction of the singlet excited state of CDs.

Next, based on the time-correlated-single-photon-counting method, the fluorescence emission decay profiles for $\mathrm{CDs}^{-} 4$ were acquired (Figure $4 a$ ). The analysis of the decay profiles at $460 \mathrm{~nm}$ (excitation at $376 \mathrm{~nm}$ ) for the singlet excited state of $\mathrm{CDs}^{-} 4$ was exclusively monoexponentially fitted with a lifetime of 14.0 ns. Addition of the positively charged $\mathrm{MoS}_{2}$-based material 3 to the negatively charged $\mathrm{CDs}^{-} 4$ resulted in biexponential fitting, giving rise to the identification of two components, namely, one with the same lifetime, attributed to non-interacting CDs and a faster new one with 3.0 ns lifetime, corresponding to the fluorescence quenching of the emission intensity of the singlet excited state of CDs within the CDs/MoS ensembles. By comparing the lifetime of unbound CDs with the one attributed to the $\mathrm{CDs} / \mathrm{MoS}_{2}$ ensembles, the quenching rate constant $k_{\mathrm{q}}^{\mathrm{S}}$ and the quenching quantum yield $\Phi_{\mathrm{q}}^{\mathrm{s}}$ were estimated to be $2.67 \times 10^{8} \mathrm{~s}^{-1}$ and 0.79 , respectively. As a consequence, the photoluminescence ratio $\mathrm{I}_{0} / \mathrm{l}$ shows a linear trend for the addition of $\mathbf{2}$ to $\mathrm{CDs}^{-} \mathbf{4}$ and a faster as well as nonlinear trend for the addition of 3 to $\mathrm{CDs}^{-} \mathbf{4}$ (Figure $4 \mathrm{~b}$ ), implying that in the latter an additional process takes place, namely, the electrostatic formation of the $\mathrm{CDs} / \mathrm{MoS}_{2}$ ensemble. This is further confirmed upon examination of the fluorescence decay profile for the blank measurement employing neutral $\mathrm{MoS}_{2}$ based derivative 2 , which remained monoexponentially fitted, following the lifetime of intact $\mathrm{CDs}^{-} 4$ (Figure 4c).

Finally, the electrocatalytic activity of $\mathrm{CDs} / \mathrm{MoS}_{2}$ towards the hydrogen evolution reaction (HER) was examined by employing a rotating disc working glassy carbon electrode in a standard three-electrode glass cell at a scan rate of $5 \mathrm{mV} / \mathrm{sec}$ in $0.5 \mathrm{M} \mathrm{H}_{2} \mathrm{SO}_{4}$. In general, $\mathrm{MoS}_{2}$ are promising materials for HER, based on the overpotential and Tafel slope values they exhibit. ${ }^{[11}$, ${ }^{22]}$ In addition, the aqueous solubility of CDs together with the presence of surface functional groups contribute to draw hydrated protons, thus enhancing proton adsorption capacity, ${ }^{[23]}$ Based on the above and considering that hydrogen binds too strongly to $\mathrm{S}$, hence leaving as primary active site for $\mathrm{MoS}_{2}$ the Mo edge, the performance of $\mathrm{CDs} / \mathrm{MoS}_{2}$ towards the HER was probed by linear sweep voltammetry. The polarization curve of $\mathrm{CDs} / \mathrm{MoS}_{2}$ along with those of individual $\mathrm{CDs}^{-} 4$ and bare glassy carbon electrode for comparison are shown in Figure $5 \mathrm{a}$. For a 
(a)

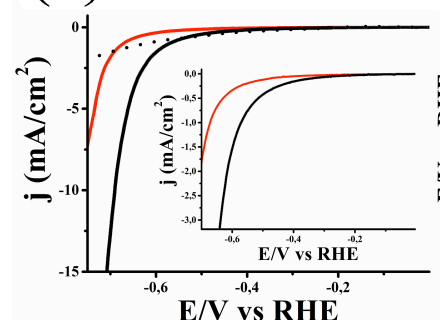

(b)

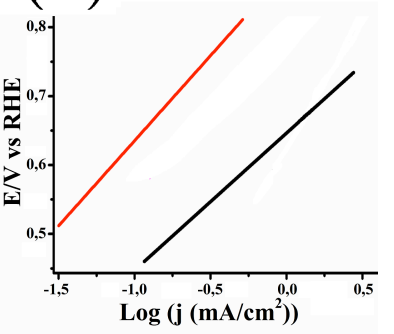

Figure 5. (a) Linear sweep voltammograms for the HER of $\mathrm{CDs} / \mathrm{MoS}_{2}$ (black), individual $\mathrm{CDs}^{-} 4$ (red) and bare glassy carbon electrode (dotted). Inset: enlarged region near the onset. (b) Tafel plots for CDs/MoS ${ }_{2}$ (black) and individual $\mathrm{CDs}^{-} 4$ (red) showing overpotential vs current density.

given potential, the cathodic current increased for $\mathrm{CDs} / \mathrm{MoS}_{2}$ as compared to that based on individual $\mathrm{CDs}^{-} \mathbf{4}$ and the bare carbon glassy electrode. The evolution of gaseous hydrogen for $\mathrm{CDs} / \mathrm{MoS}_{2}$ was visualized as bubbles appearing at currents as small as $0.5 \mathrm{~mA} / \mathrm{cm}^{2}$, with enhanced rate at around $-0.7 \mathrm{~V}$ vs RHE. Since the cathodic current density is proportional to the amount of hydrogen evolved, the latter result highlights the better catalytic activity for $\mathrm{CDs} / \mathrm{MoS}_{2}$ and prominent hydrogen evolution behavior exhibiting an onset overpotential near $-0.5 \mathrm{~V}$ vs $\mathrm{RHE}$, which is lower than the individual $\mathrm{CDs}^{-} 4$ by around 150 $\mathrm{mV}$. The better electrocatalytic activity of $\mathrm{CDs} / \mathrm{MoS}_{2}$ is mainly attributed to a synergic effect due to enhanced charge-transfer kinetics owed to the intimate contact between the two species CDs and $\mathrm{MoS}_{2}$ as well as the presence of active sites in $\mathrm{MoS}_{2}$ Next, the linear regions of the Tafel plots (Figure $5 b$ ) were fit to the Tafel equation, $\eta=B \log j+a$, where $\eta$ is the overpotential, $j$ is the current density and $B$ is the Tafel slope, to further characterize the fluent charge transport efficiency and the efficacy of the electrocatalytic reaction. Analysis of the Tafel slope helps to elucidate the possible HER mechanism and define the rate-limiting step. The Tafel slope for $\mathrm{CDs} / \mathrm{MoS}_{2}$ ensemble was found to be $22 \mathrm{mV} / \mathrm{dec}$, smaller than that owed to $\mathrm{CDs}^{-} 4$ by $4 \mathrm{mV} / \mathrm{dec}$. Considering that smaller Tafel slope suggests that for the generation of an equivalent current only a lower overpotential is needed to apply, the electrocatalytic activity of individual $\mathrm{CDs}^{-} 4$ is improved upon realization of the $\mathrm{CDs} / \mathrm{MoS}_{2}$ ensemble. The latter improvement in charge transport is attributed to good electrical contact between the two components in the donor-acceptor $\mathrm{CDs} / \mathrm{MoS}_{2}$ ensemble, in which charge-transfer phenomena prevail. Moreover, the small Tafel slope of $\mathrm{CDs} / \mathrm{MoS}_{2}$ manifests that the electrochemical desorption of adsorbed hydrogen atoms onto the modified electrode to generate hydrogen is the rate-limiting step - see equations 2 and 3 below. Based on the widely applied mechanisms for the HER, initially a proton is adsorbed onto the electrode surface via a reduction process (Volmer adsorption [Eq. (1)]) followed by either direct bonding of the adsorbed hydrogen atom with another proton and electron transfer from the electrode surface (Heyrovsky desorption [Eq. (2)]) or recombination of two hydrogen atoms adsorbed on the electrode surface (Tafel desorption [Eq. (3)]).
Volmer adsorption: $\mathrm{H}^{+}+\mathrm{e}^{-} \rightarrow \mathrm{H}^{-}$(ads) Heyrovsky desorption: $\mathrm{H}_{(\text {ads })}+\mathrm{H}^{+}+\mathrm{e}^{-} \rightarrow \mathrm{H}_{2}$ Tafel desorption: $\mathrm{H}_{\text {(ads) }}+\mathrm{H}^{\circ}$ (ads) $\rightarrow \mathrm{H}_{2}$

\section{Conclusions}

We developed aqueous stable CDs/MoS ${ }_{2}$ ensembles, in which the two components are tightly held together in a controlled fashion by electrostatic interactions. Electronic absorption and photoluminescence titration assays, complemented by timeresolved fluorescence emission, unambiguously proved the formation of the ensembles and the electronic interplay within them. Significant quenching of the CDs centred photoluminescence by $\mathrm{MoS}_{2}$ was revealed, prompting to an additional deactivation channel - electron and/or energy transfer - starting from the singlet excited state of CDs within the $\mathrm{CDs} / \mathrm{MoS}_{2}$ ensembles. Moreover, the electrocatalytic performance of $\mathrm{CDs} / \mathrm{MoS}_{2}$ was evaluated regarding the HER and found improved in comparison with that of the individual CDs species. Without a doubt, such $\mathrm{CDs} / \mathrm{MoS}_{2}$ ensembles performing in electron donor-acceptor schemes can be further exploited for managing charge-transfer processes as well as for electrocatalysis and may be useful for advancing the field of energy conversion in a wide range of technological and environmental applications.

\section{Experimental Section}

Instrumentation. Steady-state UV-Vis electronic absorption spectra were recorded on a PerkinElmer (Lambda 19) UV-Vis-NIR spectrophotometer. Steady-state emission spectra were recorded on a Fluorolog-3 JobinYvon-Spex spectrofluorometer (model GL3-21). Picosecond time-resolved fluorescence spectra were measured by the time-correlated-single-photon-counting (TCSPC) method on a Nano-Log spectrofluorometer (Horiba JobinYvon), by using a laser diode as an excitation source (NanoLED, $375 \mathrm{~nm}$ ) and a UV-Vis detector TBX-PMT series $(250-850 \mathrm{~nm})$ by Horiba JobinYvon. Lifetimes were evaluated with the DAS6 Fluorescence-Decay Analysis Software. Mid-infrared spectra in the region $500-4500 \mathrm{~cm}^{-1}$ were obtained on a Fourier transform IR spectrometer (Equinox 55 from Bruker Optics) equipped with a single reflection diamond ATR accessory (DuraSamp1IR II by SensIR Technologies). A drop of the solution was placed on the diamond surface, followed by evaporation of the solvent, in a stream of nitrogen, before recording the spectrum. Typically, 100 scans were acquired at $2 \mathrm{~cm}^{-1}$ resolution. Micro-Raman scattering measurements were performed at room temperature in the backscattering geometry using a RENISHAW inVia Raman microscope equipped with a CCD camera and a Leica microscope. A 2400 lines $\mathrm{mm}^{-1}$ grating was used for all measurements, providing a spectral resolution of $\pm 1 \mathrm{~cm}^{-1}$. As an excitation source the $\mathrm{Ar}^{+}$ laser (514 $\mathrm{nm}$ with less than $0,092 \mathrm{~mW}$ laser power) was used. Measurements were taken with 15 seconds of exposure times at varying numbers of accumulations. The laser spot was focused on the sample surface using a long working distance $50 x$ objective. Raman spectra were collected on numerous spots on the sample and recorded with Peltier cooled CCD camera. The data were collected and analyzed with Renishaw Wire and Origin software. Thermogravimetric analysis was performed using a TGA Q500 V20.2 Build 27 instrument by TA in a nitrogen (purity $>99.999 \%$ ) inert atmosphere. The microwave-assisted 
reaction was performed in a CEM Discover SP reactor employed in open-batch modality. Atomic force microscopy (AFM) images were acquired in air under ambient conditions using a MultiMode AFM with NanoScope $V$ controller (Bruker Nano Surfaces Division, Santa Barbara, CA) operating in tapping mode with Si tips Bruker AFM probe RTESPA PART MPP-11120-10. Samples were prepared on mica substrates. Particle height distribution analysis was carried out by using the Nanoscope Analysis Version 1.5 software (Veeco Ins). Elemental analyses were performed in a Thermo Flash EA 1112 instrument with $~ 3$ $\mathrm{mg}$ of powder samples. The DLS measurements were recorded on a Malvern Nano Zetasizer $\mathrm{HT}$, on a $10 \mathrm{~mm}$ path-length plastic cuvette. ${ }^{1} \mathrm{H}$ and ${ }^{13} \mathrm{C}$ NMR spectra were recorded in $\mathrm{D}_{2} \mathrm{O}$ solutions at $25{ }^{\circ} \mathrm{C}$ on a Bruker AV500 spectrometer. Electrochemical measurements were carried out at room temperature in $\mathrm{N}_{2}$-saturated $0.5 \mathrm{M} \mathrm{H}_{2} \mathrm{SO}_{4}$ in a standard three-compartment electrochemical cell using an EG\&G Princeton Applied Research potensiostat/galvanostat (Model PARSTAT ${ }^{R}$ 2273A). As counter electrode, a platinum wire was used and as reference a $\mathrm{Hg} / \mathrm{HgSO}_{4}\left(0.5 \mathrm{M} \mathrm{K}_{2} \mathrm{SO}_{4}\right)$ electrode was placed into Luggin capillary. The working electrode was a glassy carbon disk with geometric surface area of $0.071 \mathrm{~cm}^{2}$. Linear sweep voltammetry measurements were conducted with a scan rate of $5 \mathrm{mV} \mathrm{s}^{-1}$.

Synthesis of carbon dots. $2.0 \mathrm{~g}$ of citric acid monohydrate $(9.5 \mathrm{mmol})$ were dissolved in $16 \mathrm{~mL}$ of ultrapure water. Upon addition of $0.64 \mathrm{~mL}$ of ethylenediamine $(9.5 \mathrm{mmol})$ the solution was heated up through microwave irradiation. The mixture was irradiated in order to keep the temperature at $140^{\circ} \mathrm{C}$ for 6 minutes, after that the irradiation was stopped This yields in a yellow, transparent solid product, highly soluble in water. The product was dissolved in ultrapure water and dialyzed against ultrapure water (MWCO $=0.5-1.0 \mathrm{KDa}, 3$ days, twice a day). The dry material $\mathrm{CDs}^{-} 4$ was obtained by freeze-drying, with a yield in mass of 22 wt. \%.

Preparation of $\mathrm{MoS}_{2}$-based materials 2 and 3 . In a round bottom flask, exfoliated $\mathrm{MoS}_{2}(35 \mathrm{mg})$ and 1,2-dithiolane derivative $1(15 \mathrm{mg})$ in DMF $(10 \mathrm{~mL})$ were stirred at $70{ }^{\circ} \mathrm{C}$ for 40 hours. After that period, the reaction mixture was filtered through a PTFE membrane $(0.2 \mu \mathrm{m}$ pore size), the solid residue was extensively washed with DMF and dichloromethane to obtain material 2. Then, $15 \mathrm{mg}$ of $\mathbf{2}$ were redisperse in dichloromethane and treated with gaseous $\mathrm{HCl}$ for 2 minutes. The reaction mixture was left under stirring for 12 hours and then filtered through a PTFE membrane $(0.2 \mu \mathrm{m}$ pore size). The solid residue was extensively washed with DMF and dichloromethane to obtain the ammonium derivatized $\mathrm{MoS}_{2}$-based material 3

\section{Acknowledgements}

This project has received funding from the European Union's Horizon 2020 research and innovation programme under the Marie Sklodowska-Curie grant agreement $N^{\circ} 642742$.

Keywords: $\mathrm{MoS}_{2} \cdot$ carbon dots $\cdot$ donor-acceptor $\cdot$ hydrogen evolution reaction $\cdot$ energy conversion

[1] (a) X. Huang, Z. Zenga, H. Zhang, Chem. Soc. Rev. 2013, 42, 1934; (b) M. Pumera, Z. Sofer, A. Ambrosi, J. Mater. Chem. A 2014, 2, 8981.

[2] G. Pagona, C. Bittencourt, R. Arenal, N. Tagmatarchis, Chem. Commun. 2015, 51, 12950 .

[3] (a) J. Zheng, H. Zhang, S. Dong, Y. Liu, C. T. Nai, H. S. Shin, H. Y. Jeong, B. Liu, K. P. Loh, Nature Commun. 2014, 5, 2995; (b) Y. Yao, L. Tolentino, Z. Yang, X. Song, W. Zhang, Y. Chen, C.-P. Wong, Adv.
Funct. Mater. 2013, 23, 3577; (c) G. Eda, H. Yamaguchi, D. Voiry, T. Fujita, M. Chen, M. Chhowalla, Nano Lett. 2011, 11, 5111; (d) Z. Zeng, Z. Yin, X. Huang, H. Li, Q. He, G. Lu, F. Boey, H. Zhang, Angew. Chem. Int. Ed. 2011, 50, 11093.

[4] (a) D. Voiry, A. Goswami, R. Kappera, C. d. C. C. Silva, D. Kaplan, T. Fujita, M. Chen, T. Asefa, M. Chhowalla, Nature Chem. 2015, 7, 45; (b) K. C. Knirsch, N. C Berner, H. C. Nerl, C. S. Cucinotta, Z. Gholamvand, N. McEvoy, Z. Wang, I. Abramovic, P. Vecera, M. Halik, S. Sanvito, G. S. Duesberg, V. Nicolosi, F. Hauke, A. Hirsch, J. N. Coleman, C. Backes, ACS Nano 2015, 9, 6018.

[5] (a) S. S. Chou, M. De, J. Kim, S. Byun, C. Dykstra, J. Yu, J. Huang, V. P. Dravid, J. Am. Chem. Soc. 2013, 135, 4584; (b) L. Zhou, B. He, Y. Yang, Y. He, RSC Adv. 2014, 4, 32570; (c) R. Anbazhagan, H.-J. Wang, H.-C. Tsai, R.-J. Jeng, RSC Advances 2014, 4, 42936; (d) J.-S. Kim, H.-W. Yoo, H. O. Choi, H.-T. Jung, Nano Lett. 2014, 14, 5941; (e) T. Liu, S. Shi, C. Liang, S. Shen, L. Cheng, C. Wang, X. Song, S. Goel, T. E. Barnhart, W. Cai, Z. Liu, ACS Nano 2015, 9, 950; (f) E. P. Nguyen, B. J. Carey, J. Z. Ou, J. van Embden, E. D. Gaspera, A. F. Chrimes, M. J. S. Spencer, S. Zhuiykov, K. Kalantar-zadeh, T. Daeneke, Adv. Mater. 2015, 27, 6224; (g) K. Cho, M. Min, T.-Y. Kim, H. Jeong, J. Pak, J.-K. Kim, J. Jang, S. J. Yun, Y. H. Lee, W.-K. Hong, T. Lee, ACS Nano 2015 , $9,8044$.

[6] X. Chen, N. C. Berner, C. Backes, G. S. Duesberg, A. R. McDonald, Angew. Chem. Int. Ed. 2016, 55, 5803.

[7] R. Canton-Vitoria, Y Sayed-Ahmad-Baraza, M. Pelaez-Fernandez, R. Arenal, C. Bittencourt, C. P. Ewels, N. Tagmatarchis, NPJ 2D Mater. Appl. 2017, 1, 13.

[8] X. Xu, R. Ray, Y. Gu, H. J. Ploehn, L. Gearheart, K. Raker, W. A. Scrivens, J. Am. Chem. Soc. 2004, 126, 12736.

[9] J. Gu, D. Hu, J. Huang, X. Huang, Q. Zhang, X. Jia, K. Xi, Nanoscale 2016, 8, 3973

[10] (a) A. N. Emam, S. A. Loutfy, A. A. Mostafa, H. Awad, M. B. Mohamed, RSC Advances 2017, 7, 23502; (b) Y. Sun, W. Cao, S. Li, S. Jin, K. Hu, L. Hu, Y. Huang, X. Gao, Y. Wu, X. J. Liang, Sci. Rep. 2013, 3, 3036.

[11] K. K. R. Datta, G. Qi, R. Zboril, E. P. Giannelis, J. Mater. Chem. C 2016, 4, 9798.

[12] S. N. Baker, G. A. Baker, Angew. Chem. Int. Ed. 2010, 49, 6726.

[13] (a) B. C. M. Martindale, E. Joliat, C. Bachmann, R. Alberto, E. Reisner, Angew. Chem. Int. Ed. 2016, 55, 9402; (b) J. T. Margraf, F. Lodermeyer, V. Strauss, P. Haines, J. Walter, W. Peukert, R. D. Costa, T. Clark, D. M. Guldi, Nanoscale Horiz. 2016, 1, 220; (c) X. Li, M. Rui, J. Song, Z. Shen, H. Zeng, Adv. Funct. Mater. 2015, 25, 4929; (d) B. C. M. Martindale, G. A. M. Hutton, C. A. Caputo, E. Reisner, J. Am. Chem. Soc. 2015, 137, 6018; (e) W. Kwon, G. Lee, S. Do, T. Joo, S. W. Rhee, Small 2014, 10, 506.

[14] (a) V. Strauss, J. T. Margraf, T. Clark, D. M. Guldi, Chem. Sci. 2015, 6, 6878; (b) T. Skaltsas, A. Stergiou, D. D. Chronopoulos, S. Zhao, H. Shinohara, N. Tagmatarchis, J. Phys. Chem. C 2016, 120, 8550.

[15] P. Yu, X. Wen, Y.-R. Toh, Y.-C. Lee, K.-Y. Huang, S. Huang, S. Shrestha, G. Conibeer, J. Tang, J. Mater. Chem. C 2014, 2, 2894.

[16] V. Strauss, J. T. Margraf, K. Dirian, Z. Syrgiannis, M. Prato, C. Wessendorf, A. Hirsch, T. Clark, D. M. Guldi, Angew. Chem. Int. Ed. 2015, 54, 8292.

[17] F. Arcoudi, V. Strauss, L. Dordevic, A. Cadranel, D. M. Guldi, M. Prato, Angew. Chem. Int. Ed. 2017, 56, 12097.

[18] (a) Y. Wang, A. Hu, J. Mater. Chem. C 2014, 2, 6921; (b) Y. Du, S. Guo, Nanoscale 2016, 8, 2532.

[19] C. Backes, R. J. Smith, N. McEvoy, N. C. Berner, D. McCloskey, H. C. Nerl, A. O'Neill, P. J. King, T. Higgins, D. Hanlon, N. Scheuschner, J. Maultzsch, L. Houben, G. S. Duesberg, J. F. Donogan, V. Nicolosi, J. N. Coleman, Nat. Comm. 2014 5, 4576.

[20] (a) H. Li, Q. Zhang, C. C. R. Yap, B. K. Tay, T. H. T. Edwin, A. Olivier, D. Baillargeat, Adv. Funct. Mater. 2012, 22, 1385; (b) S.-L. Li, H. Miyazaki, H. Song, H. Kuramochi, S. Nakaharai, K. Tsukagoshi, ACS Nano 2012, 6, 7381. 
[21] X. Chia, A. Y. S. Eng, A. Ambrosi, S. M. Tan, M. Pumera, Chem. Rev 2015, 115, 11941

[22] (a) T. F. Jaramillo, K. P. Jørgensen, J. Bonde, J. H. Nielsen, S. Horch, I. Chorkendorff, Science 2007, 317, 100; (b) J. Kibsgaard, Z. Chen, B. N Reinecke, T. F. Jaramillo, Nature Mater. 2012, 11, 963; (c) Y. Li, H. Wang, L. Xie, Y. Liang, G. Hong, H. Dai, J. Am. Chem. Soc. 2011, 133,
7296; (d) D. Voiry, M. Salehi, R. Silva, T. Fujita, M. Chen, T. Asefa, V. B. Shenoy, G. Eda, M. Chhowalla, NanoLett. 2013, 13, 6222.

[23] H. Li, J. Liu, S. J. Guo, Y. L. Zhang, H. Huang, Y. Liu and Z. H. Kang, J. Mater. Chem. B 2015, 3, 2378. 


\section{Entry for the Table of Contents}

\section{FULL PAPER}

Aqueous stable CDs/MoS 2 ensembles were developed by electrostatic interactions. The efficient photoluminescence quenching of $\mathrm{CDs}$ by $\mathrm{MoS}_{2}$ together with the improved electrocatalytic performance of $\mathrm{CDs} / \mathrm{MoS}_{2}$ towards the HER will be employed to advance the field of energy conversion applications.

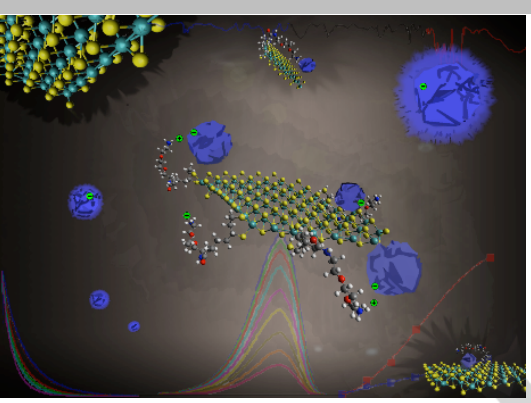

Ruben Canton-Vitoria ${ }^{[a] \#, ~ L o r e n z o ~}$ Vallan $^{[b] \#}$, Esteban Urriolabeitía ${ }^{[c]}$, Ana M. Benito ${ }^{[b]}$, Wolfgang K. Maser ${ }^{[b]}$, Nikos Tagmatarchis*aa

Page No. - Page No.

Electronic interactions in illuminated carbon dots $/ \mathrm{MoS}_{2}$ ensembles and electrocatalytic activity towards hydrogen evolution 